\title{
The Security of the FDH Variant of Chaum's Undeniable Signature Scheme
}

\author{
Wakaha Ogata $^{1}$ and Kaoru Kurosawa ${ }^{2}$ and Swee-Huay Heng ${ }^{3}$ \\ 1 Tokyo Institute of Technology, \\ 2-12-1 O-okayama, Meguro-ku,Tokyo, 152-8552 Japan \\ wakaha@craft.titech.ac.jp \\ 2 Department of Computer and Information Sciences, \\ Ibaraki University, \\ 4-12-1 Nakanarusawa, Hitachi, Ibaraki 316-8511, Japan \\ kurosawa@cis.ibaraki.ac.jp \\ 3 Multimedia University, \\ Jalan Ayer Keroh Lama, 75450 Melaka, Malaysia \\ shheng@mmu . edu . my
}

\begin{abstract}
In this paper, we first introduce a new kind of adversarial goal called forge-and-impersonate in undeniable signature schemes. Note that forgeability does not necessarily imply impersonation ability. We then classify the security of the FDH variant of Chaum's undeniable signature scheme according to three dimensions, the goal of adversaries, the attacks and the ZK level of confirmation and disavowal protocols. We finally relate each security to some well-known computational problem. In particular, we prove that the security of the FDH variant of Chaum's scheme with NIZK confirmation and disavowal protocols is equivalent to the $\mathrm{CDH}$ problem, as opposed to the GDH problem as claimed by Okamoto and Pointcheval.
\end{abstract}

Keywords: Undeniable signature, security analysis

\section{Introduction}

\section{$1.1 \quad$ Background}

The notion of undeniable signature schemes was introduced by Chaum and van Antwerpen in 1989 [11]. Since then, there have been a wide range of research covering a variety of different schemes for undeniable signatures. The validity or invalidity of an undeniable signature can only be verified with the signer's consent by engaging interactively or non-interactively in a confirmation or disavowal protocol respectively, as opposed to a digital signature in which its validity is universally verifiable. Extended schemes possess variable degrees of security and additional features such as convertibility $[6,15,23]$, designated-verifier technique [21], designated-confirmer technique [9], and so on. Among others, we also include $[8,12,19,18,17]$. 
Undeniable signatures have various applications in cryptography such as in licensing softwares, electronic voting and auctions. The most popular application is in licensing softwares. For example, software vendors might want to sign on their products to provide authenticity to their paying customers. Nevertheless, they strictly disallow dishonest users who have illegally duplicated their softwares to verify the validity of these signatures. Undeniable signature scheme plays an important role here as it allows only legitimate users to verify the validity of the signatures on the softwares.

The first proposal of undeniable signature which is based on the intractability of the computational Diffie-Hellman (CDH) problem was due to Chaum and van Antwerpen [11] and it was further improved by Chaum [8]. It is a simple and nice scheme.

On the other hand, in general, each undeniable signature scheme may have three variants of confirmation and disavowal protocols, namely, the perfect zeroknowledge protocol (ZKIP), the 3-move honest-verifier zero-knowledge protocol (HVZK) and the non-interactive zero-knowledge protocol (NIZK) with designatedverifier technique.

However, the unforgeability of Chaum's undeniable signature scheme (under any types of confirmation and disavowal protocols) has been an open problem for a long time. Recently, Okamoto and Pointcheval [25] proved the security of the full-domain hash (FDH) [5,13] variant of Chaum's scheme with NIZK confirmation and disavowal protocols. They proved that its security is equivalent to the gap Diffie-Hellman (GDH) problem in the random oracle model, where one is allowed to use the decisional Diffie-Hellman (DDH) oracle to solve the $\mathrm{CDH}$ problem.

\subsection{Our Contributions}

In this paper, we first introduce a new kind of adversarial goal called forge-andimpersonate in undeniable signature schemes. In the past, the main adversarial goal is forging and thus the most desirable security notion is the security against existentially forgery under adaptive chosen message attack [20]. In the new adversary model, the adversary not only attempts to forge but it also attempts to impersonate a legitimate signer. More precisely, an adversary first forges a message-signature pair and next executes a confirmation protocol with a verifier, trying to convince the verifier that the signature is indeed valid. Note that forgeability does not necessarily imply impersonation ability.

We then classify the security of the FDH variant of Chaum's undeniable signature scheme according to three dimensions, the adversarial goals, the attacks and the ZK level of confirmation and disavowal protocols. Finally, we prove the equivalence between each security and some well-known computational problem under various types of confirmation and disavowal protocols as shown in Table 1. However, we cannot solve the three cells marked "?" and it will be a further work to make them clear.

In our result, we also point out that the claim of Okamoto and Pointcheval as mentioned at the end of Section 1.1 is wrong. Following our result from Theorem 
Table 1. The Equivalence

\begin{tabular}{|c||c|c||c|c|}
\hline \multicolumn{1}{|c||}{} & \multicolumn{2}{c||}{ forge (F) } & \multicolumn{2}{c|}{ forge-and-impersonate (FI) } \\
\cline { 2 - 5 } & passive & active & passive & active \\
\hline \hline ZKIP & $\begin{array}{c}\text { CDH } \\
\text { (Theorem 2) }\end{array}$ & $?$ & $?$ \\
\hline HVZK & $\begin{array}{c}\text { CDH } \\
(\text { Theorem 3) }\end{array}$ & $?$ & $\begin{array}{c}\text { DLOG } \\
\text { (Theorem 4) }\end{array}$ & $\begin{array}{c}\text { one-more DLOG } \\
\text { (Theorem 6) }\end{array}$ \\
\hline NIZK & $\begin{array}{c}\text { CDH } \\
\text { (Theorem 1) }\end{array}$ & - & $\begin{array}{c}\text { DLOG or break PKS } \\
\text { (Theorem 5) }\end{array}$ & - \\
\hline \multicolumn{3}{|c|}{ *PKS denotes the verifier's public key system } \\
\hline
\end{tabular}

1 which is indicated in Table 1, we show that the unforgeability of the FDH variant of Chaum's scheme with NIZK confirmation and disavowal protocols is equivalent to the $\mathrm{CDH}$ problem, as opposed to the GDH problem as claimed by them (cf. Claim 1). Further comments on their flaw will be given in Section 3.1.

Following is some explanation on Table 1 . In the passive attack, the adversary does not interact with the prover. What the adversary does is eavesdropping and she is in possession of transcripts of conversations between the prover and the verifier. In the active attack, the adversary gets to play the role of a cheating verifier, interacting with the prover several times, in an effort to extract some useful information before the forgery or forge-and-impersonate attempt. We remark that if the scheme employs the NIZK confirmation and disavowal protocols then it is not necessary to consider the active attack.

Meanwhile, there exists another security notion for undeniable signatures called invisibility which was first introduced by Chaum et al. [12]. This notion is essentially the inability to determine whether a given message-signature pair is valid for a given user. We can prove the invisibility of the FDH variant of Chaum's scheme and show the similar result as in Table 1.

\subsection{Organization}

The remainder of this paper is organized as follows. In Section 2, we recall the definitions for some computational problems and the definition for undeniable signatures. We also describe the FDH variant of Chaum's scheme and all the confirmation and disavowal protocols associated with it. In Section 3, we explore the unforgeability of the FDH variant of Chaum's scheme with NIZK protocols. In particular, we point out the flaw in Okamoto and Pointcheval's claim in Section 3.1 and provide a correct formal proof in Section 3.2. In Section 4, we present a new adversary model for undeniable signatures. In Section 5, we analyze and discuss the security of the FDH variant of Chaum's scheme under various confirmation and disavowal protocols comprehensively. Finally, we conclude this paper in Section 6. 


\section{Preliminaries}

\subsection{Some Computational Problems}

Let $G$ be an Abelian group of prime order $q$, and let $g$ be a generator of $G$. We say that $\left(g, g^{x}, g^{r}, g^{z}\right)$ is a DH-tuple if $z=x r \bmod q$.

The DDH problem is to decide if $\left(g, g^{x}, g^{r}, g^{z}\right)$ is a DH-tuple. The CDH problem is to compute $g^{x r}$ from $\left(g, g^{x}, g^{r}\right)$. The GDH problem is to solve the $\mathrm{CDH}$ problem with the help of a DDH oracle. (Informally, it means that the $\mathrm{CDH}$ problem is hard but the DDH problem is easy.) The DLOG problem is to compute $x$ from $g^{x}$.

We also briefly define the one-more DLOG problem as follows [3, 4]:

A one-more DLOG adversary is a randomized, polynomial time algorithm $M$ that gets input $g$ and has access to two oracles, namely, a DLOG oracle that given $y \in G$ returns $x \in Z_{q}$ such that $g^{x}=y$, and a challenge oracle that each time it is invoked (it takes no inputs), returns a random challenge point $y \in G$. We say that the adversary $M$ wins if for arbitrary (polynomially bounded) $t$ challenge oracle access, it can find the DLOGs of all the challenges with at most $t-1$ (strictly less than $t$ ) DLOG oracle access.

\subsection{Undeniable Signatures}

We briefly review the concept of undeniable signatures introduced by Chaum and van Antwerpen [11].

Definition 1. An undeniable signature scheme consists of the following two polynomial time algorithms and two possibly interactive polynomial time protocols (note that in some schemes confirmation and disavowal protocols can be combined as a single protocol and they are usually zero-knowledge protocols).

- Key Generation. On input the security parameter $1^{k}$, the algorithm produces a pair of matching public and secret keys $(p k, s k)$.

- Signing. On input a secret key sk and a message $m$, the algorithm returns a signature $\sigma$.

- Confirmation Protocol. A protocol between a signer and a verifier such that when given a message $m$, a signature $\sigma$ and a public key pk, allows the signer to convince the verifier that $\sigma$ is indeed a valid signature on $m$ for a public key pk, with the knowledge of the secret key sk. If $(m, \sigma)$ is invalid, then no signer can prove it with non-negligible probability.

- Disavowal Protocol. A protocol between a signer and a verifier such that when given a message $m$, a signature $\sigma$ and a public key pk, allows the signer to convince the verifier that $\sigma$ is an invalid signature on $m$ for a public key $p k$, with the knowledge of the secret key sk. If $(m, \sigma)$ is valid, then no signer can prove it with non-negligible probability.

In the existing literature, the unforgeability for undeniable signatures is similar to the one for ordinary digital signatures, which is the notion of existential 
unforgeability against adaptive chosen message attack [20]. The only difference is that besides the signing oracle access, the forger of an undeniable signature is also allowed to access to the confirmation/disavowal oracle. The confirmation/disavowal oracle is simulated based on the types of attacks mounted, i.e. passive attack and active attack.

Informally speaking, the forger is given the public key, and after some adaptive signing queries and confirmation/disavowal queries, the forger attempts to produce a valid message-signature pair $(m, \sigma)$ such that $m$ has never been queried to the signing oracle and $(m, \sigma)$ has never been queried to the confirmation/disavowal oracle earlier. We say that the forger is successful if it can output such a valid forgery.

\subsection{The FDH Variant of Chaum's Undeniable Signature Scheme}

The FDH variant of Chaum's scheme is described as follows. Let $G$ be an Abelian group of prime order $q$, and let $g$ be a generator of $G$.

- Key Generation. On input the security parameter $1^{k}$, choose $x \in Z_{q}$ randomly and compute $y=g^{x}$. Choose a cryptographic hash function $H$ : $\{0,1\}^{*} \rightarrow G$. Set the public key as $(g, y, H)$ and the secret key as $x$.

- Signing. On input the public key $(g, y, H)$, the secret key $x$ and a message $m \in\{0,1\}^{*}$, the algorithm returns the signature as $\sigma=H(m)^{x}$.

- Confirmation Protocol. Given a message-signature pair $(m, \sigma)$, the signer proves that $(g, y, H(m), \sigma)$ is a DH-tuple.

- Disavowal Protocol. Given a message-signature pair $(m, \sigma)$, the signer proves that

$(g, y, H(m), \sigma)$ is not a DH-tuple.

Confirmation and Disavowal Protocols. There are various confirmation and disavowal protocols associated with Chaum's scheme, each with variable degrees of zero-knowledgeness and efficiency. We make an effort to summarize the various confirmation and disavowal protocols as follows.

Zero-Knowledge Interactive Proof (ZKIP). The first proposal by Chaum and van Antwerpen was not zero-knowledge [11]. In [8], an improved version with zero-knowledgeness was proposed. The confirmation protocol is a 4-move ZKIP for language of DH-tuples. For brevity, we describe the complete protocol in Fig. 1-(a).

A somewhat inefficient ZKIP disavowal protocol which requires more than 4-move was also proposed in [8]. A single execution of the protocol is as depicted in Fig. 1-(b). In this figure, $\operatorname{com}(s)$ denotes the commitment of $s$ and $\operatorname{decom}(s)$ denotes the revealing of $s$.

3-Move Honest-Verifier Zero-Knowledge Proof (HVZK). A 3-move honest-verifier zero-knowledge (HVZK) confirmation protocol is depicted in Fig. 2-(a). The corresponding 3-move HVZK disavowal protocol was shown by Camenisch and Shoup recently [7]. We describe the protocol in Fig. 2-(b). 


\begin{tabular}{|l|l|l|}
\hline \multicolumn{1}{|c|}{ Signer } & & \multicolumn{1}{c|}{ Verifier } \\
\hline & $\stackrel{R}{*} Z_{q}$ & $\begin{array}{l}a, b \stackrel{R}{\leftarrow} Z_{q} \\
c=g^{a} H(m)^{b}\end{array}$ \\
$z_{1}=c g^{r}$ & $\stackrel{z_{1}, z_{2}}{\rightleftarrows}$ & \\
$z_{2}=z_{1}^{x}$ & $\stackrel{a, b}{\stackrel{r}{\longrightarrow}}$ & \\
$c \stackrel{?}{=} g^{a} H(m)^{b}$ & $\stackrel{?}{\longrightarrow}$ & $\begin{array}{l}z_{1} \stackrel{?}{=} g^{a+r} H(m)^{b} \\
z_{2} \stackrel{?}{=} y^{a+r} \sigma^{b}\end{array}$ \\
\hline
\end{tabular}

(a) Confirmation protocol

\begin{tabular}{|l|l|l|}
\hline Signer & & \multicolumn{1}{|c|}{ Verifier } \\
\hline & & $s \stackrel{R}{\leftarrow}\{0,1, \ldots, k\}$ \\
$a \stackrel{R}{\leftarrow} Z_{q}$ \\
$c=g^{a} H(m)^{s}$ \\
find $s^{\prime}$ s.t. & $\stackrel{c, c^{\prime}}{\longleftarrow}$ & $c^{\prime}=y^{a} \sigma^{s}$ \\
$\left(c^{x} / c^{\prime}\right)=\left(H(m)^{x} / \sigma\right)^{s^{\prime}}$ & $\stackrel{\operatorname{com}\left(s^{\prime}\right)}{\longrightarrow}$ & \\
$c \stackrel{?}{=} g^{a} H(m)^{s^{\prime}}$ & $\stackrel{\operatorname{decom}\left(s^{\prime}\right)}{\longrightarrow}$ & \\
& & $s^{\prime} \stackrel{?}{=} s$ \\
\hline
\end{tabular}

(b) A single execution of disavowal protocol

Fig. 1. ZKIP protocols

\begin{tabular}{|c|c|c|}
\hline Signer & & Verifier \\
\hline $\begin{array}{l}r \stackrel{R}{\longleftarrow} Z_{q} \\
z_{1}=g^{r} \\
z_{2}=H(m)^{r} \\
d=r+c x \bmod q\end{array}$ & $\begin{array}{l}\stackrel{z_{1}, z_{2}}{\longrightarrow} \\
\stackrel{c}{\stackrel{d}{\longrightarrow}}\end{array}$ & $\begin{array}{l}c \stackrel{R}{\longleftarrow} Z_{q} \\
g^{d} \stackrel{?}{=} z_{1} y^{c} \\
H(m)^{d} \stackrel{?}{=} z_{2} \sigma^{c}\end{array}$ \\
\hline
\end{tabular}

(a) Confirmation protocol

\begin{tabular}{|c|c|c|}
\hline Signer & & Verifier \\
\hline $\begin{array}{l}s, r, r^{\prime} \stackrel{R}{\leftarrow} Z_{q} \\
w=\left(H(m)^{x} / \sigma\right)^{s} \\
z_{1}=g^{r} / y^{r^{\prime}} \\
z_{2}=H(m)^{r} / \sigma^{r^{\prime}} \\
d=r+c x s \bmod q \\
d^{\prime}=r^{\prime}+c s \bmod q\end{array}$ & $\begin{array}{c}\stackrel{w, z_{1}, z_{2}}{\longrightarrow} \\
\stackrel{d, d^{\prime}}{\longrightarrow}\end{array}$ & $\begin{array}{l}\quad \stackrel{?}{\neq} 1 \\
c \stackrel{R}{\leftarrow} Z_{q} \\
g^{d} / y^{d^{\prime}} \stackrel{?}{=} z_{1} \\
H(m)^{d} / \sigma^{d^{\prime}} \stackrel{?}{=} z_{2} w^{c}\end{array}$ \\
\hline
\end{tabular}

(b) Disavowal protocol

Fig. 2. HVZK protocols 
Non-Interactive Zero-Knowledge Proof (NIZK). In general, a 3-move honestverifier zero-knowledge protocol can be transformed to a more efficient noninteractive zero-knowledge (NIZK) protocol by using the Fiat-Shamir transformation $[16,1]$, where we need to employ another random oracle $H^{\prime}$. However, we cannot use the above solution as a confirmation protocol or a disavowal protocol because such NIZK proof is just an ordinary digital signature.

To overcome this problem, designated-verifier technique was introduced in [21] by Jakobsson et al. In a designated-verifier confirmation proof, the signer proves that " $(g, y, H(m), \sigma)$ is a DH-tuple" or "he knows the verifier's secret key" (the signer knows the former, but not the latter). In other words, the verifier is able to produce such a valid proof himself using his secret key. By using the designated-verifier technique, one can thereby prevent illegal copies of the proof.

Using the technique shown in [14], a designated-verifier proof can be constructed for a public-secret key pair of any well-known public key system. The obtained NIZK proof is zero-knowledge in the random oracle model.

We do not give the concrete NIZK designated-verifier confirmation and disavowal protocols since different protocols are associated with different public key systems used by the verifier.

\section{Unforgeability of NIZK Scheme}

Chaum's original scheme (which does not employ a cryptographic hash function) is not secure as it is existentially forgeable. Most precisely, it succumbed to the basic multiplicative attack: suppose that an adversary has two message-signature pairs $\left(m_{1}, \sigma_{1}\right)$ and $\left(m_{2}, \sigma_{2}\right)$, where $\sigma_{1}=m_{1}^{x}$ and $\sigma_{2}=m_{2}^{x}$. Then it is obvious that $\sigma_{1} \sigma_{2}$ is a signature of $m_{1} m_{2}$.

Okamoto and Pointcheval [25] made the first attempt to analyze the security of Chaum's scheme by incorporating the full-domain hash (FDH) technique [5, 13]. In other words, they studied the security of the FDH variant of Chaum's scheme in the random oracle model by modeling the hash function $H$ as a random oracle. ${ }^{4}$ Okamoto and Pointcheval further claimed that they have solved the more than 10 years open problem, i.e. the security of the FDH variant of Chaum's scheme with NIZK protocols is equivalent to the GDH problem.

However, we are going to disprove their claim in this section. In the sequel, we first restate their claim and point out the major flaw in their proof. We then prove that the security of the FDH variant of Chaum's scheme with NIZK protocols is in fact equivalent to the $\mathrm{CDH}$ problem, a more difficult problem than GDH.

In the NIZK scheme, the public key is $\left(g, y, H, H^{\prime}\right)$, where $H^{\prime}$ is a hash function which is used for Fiat-Shamir transformation (which transforms a 3move HVZK protocol to an NIZK proof).

\footnotetext{
${ }^{4}$ Another merit in the FDH variant is that messages may be arbitrary bit strings and do not need to be encoded as group elements as in the original scheme.
} 


\subsection{The Flaw in Okamoto and Pointcheval's Claim}

Their claim is as follows.

Claim 1. [25, Theorem 9] An existential forgery under adaptively chosen message attack for the FDH variant of Chaum's undeniable signature scheme is equivalent to the GDH problem in the random oracle model, where the confirmation and disavowal protocols are NIZK.

The correctness of the above claim was shown by proving the following [25]:

(1) If there exists an algorithm $M$ that solves the GDH problem, then one can construct a forger $F$ that manage to forge a message-signature pair by running $M$ as its subroutine.

(2) If there exists a forger $F$ that forges a message-signature pair, then one can construct an algorithm $M$ that can solve the GDH problem by running $F$ as its subroutine.

The proof of (1) is wrong. In the proof, the forger $F$ runs the algorithm $M$ as follows. At first, the forger $F$ is given the public key $\left(g, y, H, H^{\prime}\right)\left(H^{\prime}\right.$ is used to transform HVZK to a non-interactive one). $F$ then chooses $m$ randomly and runs $M$ on input $(g, y, H(m))$. If $M$ submits $\left(g, y, H\left(m^{\prime}\right), \sigma^{\prime}\right)$ to the DDH oracle, then $F$ queries to its confirmation/disavowal oracle and returns the answer to $M$. $M$ finally outputs $H(m)^{x}$ with non-negligible probability from our assumption. Therefore, $F$ can forge the signature on $m$ as $H(m)^{x}$ with non-negligible probability.

However, suppose that $M$ submits $\left(g, y, H\left(m^{\prime}\right), \sigma^{\prime}\right)$ to the DDH oracle. Then what $F$ can query to its confirmation/disavowal oracle is $\left(m^{\prime}, \sigma^{\prime}\right)$, but not $\left(H\left(m^{\prime}\right), \sigma^{\prime}\right)$. Since $F$ cannot compute $m^{\prime}$ from $H\left(m^{\prime}\right)$, so it cannot query $\left(m^{\prime}, \sigma^{\prime}\right)$. More precisely, since a prover in the confirmation/disavowal protocol takes only the message $m^{\prime}$ and its signature $\sigma^{\prime}$ as input, simulating a DDH oracle would require to inverse the hash function $H$, which is obviously impossible! Therefore, $F$ fails to simulate the DDH oracle correctly. This is indeed a critical flaw.

The proof of (2) is redundant. In the proof, the confirmation/disavowal oracle is simulated by the DDH oracle. More precisely, to decide whether the given $(m, \sigma)$ is a valid pair or not, $M$ asks $(g, y, H(m), \sigma)$ to the DDH oracle, and then simulates the confirmation/disavowal oracle by itself. However, notice that $M$ can decide the validity of $(m, \sigma)$, since it can simulate the signing oracle by itself and furthermore the signing algorithm is deterministic. Thus the DDH oracle is totally redundant here as it plays no function at all.

\subsection{Correct Equivalence}

Based on the above argument, we have indirectly proven Theorem 1, i.e. the existence of $F$ is equivalent to the existence of $M$ that solves the $\mathrm{CDH}$ problem (without the DDH oracle access). For clarity and completeness, we provide a formal proof for the theorem. 
Theorem 1. The security of the FDH variant of Chaum's undeniable signature scheme with NIZK confirmation and disavowal protocols is equivalent to the CDH problem in the random oracle model.

Proof. Firstly, we show that if there exists an algorithm $M$ that solves the CDH problem with advantage $\epsilon_{M}$, then one can construct a forger $F$ that can forge in the universal way with advantage $\epsilon_{F}$, by running $M$ as a subroutine. The forger $F$ is given the public key $\left(g, y, H, H^{\prime}\right)$ where $y=g^{x}$. For any message $m, F$ computes $h=H(m)$ and gives the triple $(g, y, h)$ as input to $M$. When $M$ outputs $h^{x}, F$ simply outputs the forgery as $\left(m, \sigma=h^{x}\right)$. It is clear that $\epsilon_{F}=\epsilon_{M}$. This completes the first half of our proof.

Secondly, we show that if there exists a forger $F$ that manage to forge with advantage $\epsilon_{F}$, then one can construct an algorithm $M$ that can solve the CDH problem with advantage $\epsilon_{M}$, by running $F$ as a subroutine. Suppose the input to $M$ is $\left(g, g^{x}, g^{r}\right)$. $M$ then starts running $F$ by feeding $F$ with the public key $\left(g, y=g^{x}, H, H^{\prime}\right)$ where $H$ and $H^{\prime}$ are random oracles that will be simulated by $M$. $M$ also simulates the signing oracle and the confirmation/disavowal oracle itself. Let $q_{S}$ and $q_{H}$ be the number of signing queries and $H$-queries that $F$ issues respectively. We assume that when $F$ makes a confirmation/disavowal query for a message-signature pair $\left(m_{i}, \sigma_{i}^{\prime}\right)$, it has already made the corresponding signing query on $m_{i}$. We also assume that when $F$ requests a signature on a message $m_{i}$, it has already made the corresponding $H$-query on $m_{i}$.

When $F$ makes a $H$-query for a message $m_{i}, M$ responds with $h_{i}=H\left(m_{i}\right)=$ $g^{\alpha_{i}}$ with probability $\delta$ and $h_{i}=H\left(m_{i}\right)=\left(g^{r}\right)^{\alpha_{i}}$ with probability $1-\delta$, where $\alpha_{i}$ is chosen randomly from $Z_{q}$ and $\delta$ is a fixed probability which will be determined later.

When $F$ makes a $H^{\prime}$-query for a new str, where str is the string that $F$ would like to know its $H^{\prime}$ value. $M$ always responds with a random number. In fact, $M$ assigns some values to $H^{\prime}(s t r)$ for some str in order to simulate the confirmation/disavowal oracle. When $F$ makes a $H^{\prime}$-query for such $s t r, M$ returns $H^{\prime}($ str $)$ to $F$.

When $F$ makes a signing query for a message $m_{i}$, if $h_{i}=g^{\alpha_{i}}$ then $M$ returns $\sigma_{i}=y^{\alpha_{i}}$ as the valid signature (since $\left.y^{\alpha_{i}}=\left(g^{x}\right)^{\alpha_{i}}=h_{i}^{x}=H\left(m_{i}\right)^{x}\right)$. Otherwise, $M$ aborts and it fails to solve the $\mathrm{CDH}$ problem.

Next, we consider the case that $F$ makes a confirmation/disavowal query for a message-signature pair $\left(m_{i}, \sigma_{i}^{\prime}\right)$. In this case, $M$ has to do in two steps. In the first step, it checks the validity of $\left(m_{i}, \sigma_{i}^{\prime}\right)$ using the signing oracle. From our assumption, $F$ has already made a signing query for $m_{i}$, and $M$ answered with a valid signature $\sigma_{i}$ with probability $\delta$ (with probability $(1-\delta) M$ aborts). Therefore, if $\sigma_{i}=\sigma_{i}^{\prime}$ then it is valid, otherwise it is invalid. Remember that the signing algorithm is deterministic. In the second step, $M$ does the following. If $\left(m_{i}, \sigma_{i}^{\prime}\right)$ is a valid pair then $M$ returns the transcript of the confirmation protocol. Otherwise, it returns the transcript of the disavowal protocol. As mentioned before, $M$ can manipulate $H^{\prime}$-oracle and thus it can generate a transcript of the confirmation or disavowal protocol. (In fact, it is possible that collision occurs for $s t r$, meaning that $s t r$ is being asked to $H^{\prime}$-oracle by $F$ earlier before $M$ assigns 
a value to $H^{\prime}(s t r)$. However, this probability is negligible and thus it will not affect the overall success probability for $M$.)

Eventually, $F$ halts and outputs a forgery $(m, \sigma)$. We assume that $F$ has queried the $H$-oracle on $m$ and so $m=m_{i}$ for some $i$. If $h_{i}=\left(g^{r}\right)^{\alpha_{i}}$, then we have $\sigma=h_{i}^{x}=\left(g^{r \alpha_{i}}\right)^{x}$. Consequently, $M$ outputs $g^{x r}=\sigma^{1 / \alpha_{i}}$ and thus it solves the $\mathrm{CDH}$ problem. Otherwise, $M$ aborts and it fails to solve the CDH problem.

To complete the proof, it remains to calculate the probability that $M$ does not abort. The probability that $M$ answers to all the signing queries is $\delta^{q S}$ and $M$ outputs $g^{x r}$ with probability $1-\delta$. Therefore, the probability that $M$ does not abort during the simulation is $\delta^{q_{S}}(1-\delta)$. This value is maximized at $\delta_{\text {opt }}=$ $1-1 /\left(q_{S}+1\right)$. This shows that $M$ 's advantage $\epsilon_{M}$ is at least $\left(1 / e\left(1+q_{S}\right)\right) \epsilon_{F}$, where $e$ is the base of the natural logarithm. This is because the value $\left(1-1 /\left(q_{S}+1\right)\right)^{q_{S}}$ approaches $1 / e$ for large $q_{S}$. This completes our proof.

\section{New Adversary Model}

In this section, we present a new adversary model for undeniable signatures that incorporates a new adversarial goal called forge-and-impersonate. In the past, the main adversarial goal is forging, i.e. one considers an undeniable signature scheme to be secure if it is existentially unforgeable against adaptive chosen message attack. In our new proposal, the adversary not only attempts to forge but it also attempts to impersonate a legitimate signer.

It is clear that forgeability does not necessarily imply impersonation ability. Hence the new adversarial goal is stronger. (On the other hand, the latter implies the former because if $(m, \sigma)$ is invalid, then any signer can convince the verifier with only negligible probability in the confirmation protocol. See Section 2.2.)

Now, we present our proposal and explain what motivates us to consider this new adversarial goal.

\subsection{Adversarial Goals}

As usual, we classify adversaries by their ultimate adversarial goals. Normally, an adversary with the motive to forge a new message-signature pair $(m, \sigma)$ is given the name forger. As mentioned earlier, this is the traditional security notion.

Now, we introduce a new type of adversary. The new adversarial goal is to forge a message-signature pair $(m, \sigma)$ and further convincing a (honest) verifier that $\sigma$ is indeed a valid signature on $m$, by executing the confirmation protocol with the verifier. To avoid confusion, we stick to the following notation. We denote the former type of adversary as forge $(\mathrm{F})$ and the latter as forge-andimpersonate $(\mathrm{FI})$.

It is pretty hard for this new adversary to gain a success, but let us look at the motivation for the adversary. As noted earlier in the introduction part, the most common application of undeniable signatures is in licensing softwares. If an adversary succeeds in forging a signature (but not in convincing the verifier by executing a confirmation protocol), no doubt it would cause some damage 
to the legitimate signer (e.g. Microsoft). On the other hand, if an adversary succeeds in forging as well as in impersonating, then it can sell its own softwares by impersonating an agent of Microsoft. In this case, it can actively earn some fast money through its wicked deed. This is the motivation behind the attack.

Intuitively, the security against a $\mathrm{FI}$ adversary is equivalent to a problem which is no easier than the problem which is equivalent to the security against a $\mathrm{F}$ adversary. We shall exemplify this with some security analyses in the next section.

On the other hand, we also remark that the security against FI does not imply unforgeability from the definitions. From the definition of Fl adversary, the adversary forges $(m, \sigma)$ and succeeds in the confirmation protocol. However, notice that there is a possibility that even if $(m, \sigma)$ is invalid, the adversary succeeds in the confirmation protocol. Hence, the security against FI adversary does not imply unforgeability. We also note that if we use a ZKIP confirmation protocol, then the security against FI adversary does imply unforgeability, due to the soundness of the ZKIP protocol.

\subsection{Types of Attacks}

We can also classify adversaries by their capabilities or types of attacks. More precisely, there exist two types of attacks, namely, passive attack and active attack. Obviously, passive attack is a weaker attack.

Both the passive and active adversaries have access to the signing oracle as well as the confirmation/disavowal oracle. The signing oracle plays the role similar to those in the ordinary signature scheme. We highlight the difference between a passive attack and an active attack below.

Whenever an adversary submits a confirmation/disavowal query $(m, \sigma)$, the oracle responds based on whether a passive attack or an active attack is mounted. In a passive attack, the confirmation/disavowal oracle first checks the validity of $(m, \sigma)$ using the signing oracle. If it is a valid pair, then the oracle returns "yes" and a transcript of confirmation protocol. Otherwise, the oracle returns "no" and a transcript of disavowal protocol. In an active attack, the confirmation/disavowal oracle first checks the validity of $(m, \sigma)$ using the signing oracle. If it is a valid pair, then the oracle returns "yes" and proceeds with the execution of the confirmation protocol with the adversary (acting as a cheating verifier). Otherwise, the oracle returns "no" and executes the disavowal protocol with the adversary accordingly.

\subsection{Formal Security Definitions}

In this section, we provide the formal security definitions by considering the two adversarial goals, namely forge (F) and forge-and-impersonate (FI) and the two types of attacks mounted by the adversary.

Definition 2 (Unforgeability). An undeniable signature scheme is said to be existential unforgeable under adaptive chosen message attack if no probabilistic 
polynomial time (PPT) forger $F$ has a non-negligible advantage in the following game:

1. Let $p k$ be the input to $F$.

2. The forger $F$ is permitted to issue a series of queries:

- Signing queries: $F$ submits a message $m$ and receives a signature $\sigma$ on $m$. (We consider adaptive queries here - subsequent queries is made based on previously obtained signatures.)

- Confirmation/disavowal queries: $F$ submits a message-signature pair $(m, \sigma)$, and the oracle responds based on whether a passive attack or an active attack is mounted.

In a passive attack, the confirmation/disavowal oracle first checks the validity of $(m, \sigma)$ using the signing oracle. If it is a valid pair, then the oracle returns "yes" and a transcript of confirmation protocol. Otherwise, the oracle returns "no" and a transcript of disavowal protocol.

In an active attack, the confirmation/disavowal oracle first checks the validity of $(m, \sigma)$ using the signing oracle. If it is a valid pair, then the oracle returns "yes" and proceeds with the execution of the confirmation protocol with the forger $F$ (acting as a cheating verifier). Otherwise, the oracle returns " $n o$ " and executes the disavowal protocol with $F$ accordingly.

3. At the end of this attack game, $F$ outputs a message-signature pair $(m, \sigma)$ such that $m$ has never been queried to the signing oracle and that $(m, \sigma)$ has never been queried to the confirmation/disavowal oracle earlier.

The forger $F$ wins the game if $\sigma$ is a valid signature on $m$. F's advantage in this game is defined to be $A d v(F)=\operatorname{Pr}[F$ wins $]$.

Definition 3 (Unforgeability-and-Unimpersonation). An undeniable signature scheme is said to be secure against forgery and impersonation under adaptive chosen message attack if no PPT adversary $A$ has a non-negligible advantage in the following game:

1. Let $p k$ be the input to $A$.

2. The adversary $A$ enters the learning phase where it performs a series of queries: signing queries and confirmation/disavowal queries as in the previous definitions (based on whether a passive attack or an active attack is mounted). At the end of this forgery phase, A outputs a forged messagesignature pair $(m, \sigma)$ such that $m$ has never been queried to the signing oracle and that $(m, \sigma)$ has never been queried to the confirmation/disavowal oracle earlier.

3. In the impersonation phase, A proceeds to execute the confirmation protocol with a verifier on input $(m, \sigma)$, trying to convince the verifier that $(m, \sigma)$ is a valid pair.

The adversary $A$ wins the game if it can convince the verifier that $(m, \sigma)$ is a valid message-signature pair. A's advantage in this game is defined to be $\operatorname{Adv}(A)=$ $\operatorname{Pr}[$ Awins $]$. 


\section{$4.4 \quad$ FI-Security in NIZK}

For undeniable signature schemes with designated-verifier NIZK proofs, we have to carefully define the security against FI attack. This is because in such scheme, besides breaking the undeniable signature scheme, an adversary can also impersonate by breaking the public key system of a verifier.

Therefore, we first specify the key generation algorithm of the public key system PKS of the target verifier. We denote the $\mathrm{FI}$ attack in this situation with $\mathrm{FI}^{\mathrm{PKS}}$ attack. We then adopt the following adversary model.

1. As usual, after making some oracle queries, the adversary $A$ outputs a forged message-signature pair $(m, \sigma)$.

2. Now, $A$ is given a public key of a verifier randomly.

3. Next, it outputs a non-interactive non-transferable confirmation transcript corresponding to the given public key.

We say that $A$ succeeds in $\mathrm{FI}^{\mathrm{PKS}}$ attack if the proof is accepted with nonnegligible probability, where the probability is taken over the key generation algorithm of PKS as well.

\section{The Equivalence}

\subsection{Our Objective}

Following from the previous section, it is thus clear that we need to consider four types of adversaries, namely, the passive $F$, the active $F$, the passive $F I$ and the active Fl.

There are various confirmation and disavowal protocols associate with the FDH variant of Chaum's scheme, namely, ZKIP, 3-move HVZK and 1-move NIZK.

We intend to explore further on the equivalence between the security of the scheme (with various confirmation and disavowal protocols) and some computational problems, under the various types of adversaries. In other words, our objective is to fill up Table 1 .

We remark that if the scheme employs the non-interactive confirmation and disavowal protocols (NIZK), then it is not necessary to consider active attack.

In what follows, a $x x x$ scheme denotes the scheme with $x x x$ confirmation and disavowal protocols, where $x x x$ is ZKIP, HVZK or NIZK.

\subsection{On F Attacks}

First of all, recall that in Theorem 1 of Section 3.1, we have shown that the passive $\mathrm{F}$ attack to the scheme with NIZK protocols is equivalent to the CDH problem.

Theorem 2. The ZKIP scheme is secure against each of passive/active $\mathrm{F}$ attack in the random oracle model if and only if the CDH problem is hard. 
Proof. The only if part is trivial. The if part can be shown almost similarly to Theorem 1 . However, notice that $M$ does not need to simulate the $H^{\prime}$-oracle here. The signing oracle, $H$-oracle and the first step of the confirmation/disavowal oracle are simulated similarly (see the proof of Theorem 1). The only difference is in the second step of the confirmation/disavowal oracle simulation. Please refer to the full version of this paper [24] for the concrete simulation of confirmation/disavowal oracle in an active attack. Intuitively, the zero-knowledge property of the protocols assures that $M$ can simulate the confirmation/disavowal oracle. Therefore, it is also clear that $M$ can simulate the confirmation/disavowal oracle in a passive attack, since passive attack is weaker than active attack.

Theorem 3. The HVZK scheme is secure against passive $\mathrm{F}$ attack in the random oracle model if and only if the CDH problem is hard.

Proof. The only if part is trivial. The if part can be shown almost similarly to Theorem 2 except in the confirmation/disavowal oracle simulation. Please refer to [24] for the concrete perfect simulation of the transcripts of confirmation/disavowal protocol.

\subsection{On Passive FI Attacks}

Theorem 4. The passive $\mathrm{FI}$ attack on the HVZK scheme is equivalent to the $D L O G$ problem in the random oracle model.

Proof. Firstly, we show that if there exists an algorithm $M$ that solves the DLOG problem, then an adversary $A$ can succeed in $\mathrm{Fl}$ attack by running $M$ as a subroutine. The adversary $A$ is given the public key $(g, y, H)$ where $y=g^{x}$. Since $A$ can obtain the secret key $x$ by feeding $y$ to algorithm $M$, it can succeed in the FI attack. This completes the first half of the proof.

Secondly, let $A$ be a passive $\mathrm{Fl}$ adversary. We show that one can construct an algorithm $M$ that can solve the DLOG problem by running $A$ as a subroutine. Suppose that the input to $M$ is $\left(g, g^{x}\right), M$ then starts running $A$ by feeding $A$ with the public key $\left(g, y=g^{x}, H\right)$, where $H$ is a random oracle that will be simulated by $M . M$ also simulates the signing oracle and the confirmation/disavowal oracle itself. We assume that when $A$ makes a confirmation/disavowal query for a message-signature pair $\left(m_{i}, \sigma_{i}^{\prime}\right)$, it has already made the corresponding signing query on $m_{i}$. We also assume that when $A$ requests a signature on a message $m_{i}$, it has already made the corresponding $H$-query on $m_{i}$.

When $A$ makes a $H$-query for a message $m_{i}, M$ responds with $h_{i}=g^{\alpha_{i}}$, where $\alpha_{i}$ is chosen randomly from $Z_{q}$. When $A$ makes a signing query for a message $m_{i}$, $M$ returns $\sigma_{i}=y^{\alpha_{i}}$ as the valid signature (since $\left.y^{\alpha_{i}}=\left(g^{x}\right)^{\alpha_{i}}=h_{i}^{x}=H\left(m_{i}\right)^{x}\right)$.

When $A$ makes a confirmation/disavowal query for a message-signature pair $\left(m_{i}, \sigma_{i}^{\prime}\right), A$ can distinguish between a valid pair and an invalid pair by checking the signing queries record. Further, $M$ can simulate the confirmation/disavowal oracle perfectly since the views of the honest-verifier zero-knowledge protocols are simulatable (see [24]). 
Eventually, $A$ outputs a forgery $(m, \sigma)$. It then proceeds to prove that $\sigma$ is indeed a valid signature by executing the confirmation protocol with the honestverifier. Since the confirmation protocol is a proof of knowledge of $x$, thus $M$ can extract $x$ by using the reset technique [2]. Please refer to [24] for the details.

The following theorem states the security of the scheme against passive FI attack when non-interactive zero-knowledge proofs are used.

Theorem 5. The passive $\mathrm{FI}^{\mathrm{PKS}}$ attack on the NIZK scheme is equivalent to "solving the DLOG problem" or "breaking PKS" in the random oracle model. Here, "breaking PKS" means that the adversary obtains the secret key corresponding to the given public key which is chosen randomly in PKS.

Proof. Consider an algorithm $M$ whose input is $((g, y), P k)$ where $y$ is a random element of $G$ and $P k$ is a randomly chosen public key in PKS. If $M$ outputs $x$ such that $y=g^{x}$ or $S k$ such that $(P k, S k)$ is a public-secret key pair in PKS, then we can say that $M$ succeeds in "solving the DLOG problem or breaking PKS". Clearly, if there exists such algorithm $M$, then an adversary $A$ can succeed in $\mathrm{FI}^{\mathrm{PKS}}$ attack by running $M$ as a subroutine. Thus the first half of the proof was shown.

Secondly, let $A$ be a passive $\mathrm{FI}^{\mathrm{PKS}}$ adversary. We show that one can construct an algorithm $M$ that can solve the DLOG problem or can break PKS by running $A$ as a subroutine. Suppose that the input to $M$ is $((g, y), P k)$. At first, $M$ starts running $A$ by feeding $A$ with the public key $\left(g, y, H, H^{\prime}\right)$. We assume that when $A$ makes a confirmation/disavowal query for a message-signature pair $\left(m_{i}, \sigma_{i}^{\prime}\right)$, it has already made the corresponding signing query on $m_{i}$. We also assume that when $A$ requests a signature on a message $m_{i}$, it has already made the corresponding $H$-query on $m_{i}$.

The simulation of the $H$-oracle and the signing oracle are the same as in the previous proof. The simulation of the $H^{\prime}$-oracle is the same as the proof of Theorem 1. The simulation of the confirmation/disavowal oracle is also almost the same as those in the proof of Theorem 1, except that now when $A$ makes a signing query for $m_{i}, M$ answered with a valid signature $\sigma$ with probability 1 .

Eventually, $A$ outputs a forgery $(m, \sigma)$ and requests a verifier's public key. $M$ then hands $P k$ to $A$. $A$ next generates a non-interactive non-transferable confirmation transcript corresponding to $P k$ and returns the transcript to $M$. After that, $M$ resets $A$. Unlike in the previous proof, $M$ has to rewind $A$ to the point that it has made the $H^{\prime}$-query for $s t r$ where $H^{\prime}(s t r)$ is used as a random challenge in the confirmation transcript. Using the same argument of forking lemma [26], if $A$ outputs a NIZK confirmation transcript with non-negligible probability, then rewinding $A$ with a different $H^{\prime}$ value will result $M$ in getting two confirmation transcripts for a common input $(m, \sigma)$, with non-negligible probability. From these two transcripts, $M$ can obtain a witness $W$. At last $M$ outputs $W$. Remember that the designated-verifier confirmation transcript is a proof of knowledge of $x$ (the signer's secret key) or the verifier's secret key $S k$. Therefore, we have $W=x$ or $W=S k$, that is, $M$ succeeds in solving the DLOG problem or breaking PKS. 
From the above theorem, if the target verifier uses ElGamal cryptosystem, then the passive FI attack on NIZK scheme is equivalent to the DLOG problem. If the target verifier uses RSA cryptosystem, then the passive FI attack on NIZK scheme is equivalent to "solving the DLOG problem" or "factoring the RSA modulus $N "[22]$.

\subsection{On Active FI Attacks}

Finally, we consider the last case, the active FI attack. In the active FI attack, the adversary has additional power, i.e. to execute confirmation and disavowal protocols interactively with the signer. $M$ plays the role of the signer in this scenario, interacting with the adversary whenever it receives a confirmation/disavowal query.

The proof of the following theorem is given in [24].

Theorem 6. The HVZK scheme is secure against active $\mathrm{FI}$ attack in the random oracle model if the one-more DLOG problem is hard.

\subsection{Discussion}

We have analyzed the security of the FDH variant of Chaum's scheme under various types of confirmation/disavowal protocols using the newly proposed adversary model. Their equivalence with some known computational problems are proven. In conclusion, the results we obtained are as summarized in Table 1, which follows from Theorem 1 to Theorem 6 .

The three cells marked "?" are still unsolved at the moment due to the following reasons. In the proofs of Theorem 4 and Theorem 5, $M$ can extract $x$ from $y=g^{x}$ because the confirmation protocol is a proof of knowledge of $x$, thus there exists a knowledge extractor for $x$. On the other hand, the perfect zero-knowledge confirmation protocol shown in Fig. 1-(a) is a proof of language and not a proof of knowledge. Therefore, it is impossible for us to construct such a knowledge extractor. This is the reason why we are unable to prove the equivalence between FI attack and and some well-known computational problem by using the same approach. May be there exist some other approaches to prove the equivalence, however we are yet to discover it at the moment.

However, we conjecture that the problem which should be equivalent to the security against passive $\mathrm{FI}$ and active $\mathrm{FI}$ attacks when ZKIP protocols are employed and the problem which should be equivalent to the security against active F attack when HVZK protocols are employed, should be no easier than the CDH problem. We anticipate the solution in the near future and we encourage more attempts on them.

There exists another security notion for undeniable signatures called invisibility which was first introduced by Chaum et al. [12]. This notion is essentially the inability to determine whether a given message-signature pair is valid for a given signer. We can prove the invisibility of the FDH variant of Chaum's scheme and show the similar results as in Table 1. Due to the space limitation, the details will be given in the final paper. 


\section{Conclusion}

In this paper, we introduced another new adversarial goal called forge-andimpersonate in undeniable signature schemes, and this leads to a new adversary model which is slightly stronger than the existing one. We also classified the security of the FDH variant of Chaum's undeniable signature scheme according to three dimensions, the attacks, the adversarial goals and the ZK level of confirmation and disavowal protocols, and then related each security to some well-known computational problem. In addition, we also pointed out the flaw in Okamoto and Pointcheval's claim, i.e. we proved that the unforgeability of the FDH variant of Chaum's scheme with NIZK confirmation and disavowal protocols is equivalent to the $\mathrm{CDH}$ problem, as opposed to the GDH problem as claimed by them.

\section{References}

1. M. Abdalla, J. An, M. Bellare and C. Namprempre. From identification to signatures via the Fiat-Shamir transform: minimizing assumptions for security and forward-security. Advances in Cryptology - EUROCRYPT '02, LNCS 2332, pp. 418-433, Springer-Verlag, 2002.

2. M. Bellare and A. Palacio. GQ and Schnorr identification schemes: proofs of security against impersonation under active and concurrent attacks. Advances in Cryptology - CRYPTO '02, LNCS 2442, pp. 162-177, Springer-Verlag, 2002.

3. M. Bellare, C. Namprempre, D. Pointcheval and M. Semanko. The power of RSA inversion oracles and the security of Chaum's RSA-based blind signature scheme. Financial Cryptography '01, LNCS 2339, pp. 319-338, Springer-Verlag, 2002.

4. M. Bellare, C. Namprempre, D. Pointcheval and M. Semanko. The one-more-RSAinversion problems and the security of Chaum's blind signature scheme. Journal of Cryptology, vol. 16, no. 3, pp. 185-215, Springer-Verlag, 2003.

5. M. Bellare and P. Rogaway. The exact security of digital signatures - how to sign with RSA and Rabin. Advances in Cryptology - EUROCRYPT '96, LNCS 1070, pp. 399-416, Springer-Verlag, 1996.

6. J. Boyar, D. Chaum, I. Damgård and T. Pedersen. Convertible undeniable signatures. Advances in Cryptology - CRYPTO '90, LNCS 537, pp. 189-208, SpringerVerlag, 1990.

7. J. Camenisch and V. Shoup. Practical verifiable encryption and decryption of discrete logarithms. Advances in Cryptology - CRYPTO '03, LNCS 2729, pp. 126-144, Springer-Verlag, 2003.

8. D. Chaum. Zero-knowledge undeniable signatures. Advances in Cryptology EUROCRYPT '90, LNCS 473, pp. 458-464, Springer-Verlag, 1990.

9. D. Chaum. Designated confirmer signatures. Advances in Cryptology - EUROCRYPT '94, LNCS 950, pp. 86-91, Springer-Verlag, 1995.

10. T. Chaum and T. P. Pedersen. Wallet databases with observers. Advances in Cryptology - CRYPTO '92, LNCS 740, pp. 89-105, Springer-Verlag, 1993.

11. D. Chaum and H. van Antwerpen. Undeniable signatures. Advances in Cryptology - CRYPTO '89, LNCS 435, pp. 212-216, Springer-Verlag, 1989.

12. D. Chaum, E. van Heijst and B. Pfitzmann. Cryptographically strong undeniable signatures, unconditionally secure for the signer. Advances in Cryptology CRYPTO '91, LNCS 576, pp. 470-484, Springer-Verlag, 1991. 
13. J. Coron. On the exact security of full domain hash. Advances in Cryptology CRYPTO '00, LNCS 1880, pp. 229-235, Springer-Verlag, 2000.

14. R. Cramer, I. Damgård and B. Schoenmakers. Proofs of partial knowledge and simplified design of witness hiding protocols. Advances in Cryptology - CRYPTO '94, LNCS 839, pp. 174-187, Springer-Verlag, 1994.

15. I. Damgård and T. Pedersen. New convertible undeniable signature schemes. Advances in Cryptology - EUROCRYPT '96, LNCS 1070, pp. 372-386, SpringerVerlag, 1996.

16. A. Fiat and A. Shamir. How to prove yourself: practical solutions to identification and signature problems. Advances in Cryptology - CRYPTO '86, LNCS 263, pp. 186-194, Springer-Verlag, 1987.

17. S. Galbraith and W. Mao. Invisibility and anonymity of undeniable and confirmer signatures. Topics in Cryptology - CT-RSA '03, LNCS 2612, pp. 80-97, Springer Verlag, 2003.

18. S. Galbraith, W. Mao and K. G. Paterson. RSA-based undeniable signatures for general moduli. Topics in Cryptology - CT-RSA '02, LNCS 2271, pp. 200-217, Springer Verlag, 2002.

19. R. Gennaro, H. Krawczyk and T. Rabin. RSA-based undeniable signatures. Advances in Cryptology - CRYPTO '97, LNCS 1294, pp. 132-149, Springer-Verlag, 1997.

20. S. Goldwasser, S. Micali and R. Rivest. A digital signature scheme secure against adaptative chosen-message attacks. SIAM Journal of Computing, vol. 17, no. 2, pp. 281-308, 1988.

21. M. Jakobsson, K. Sako and R. Impagliazzo. Designated verifier proofs and their applications. Advances in Cryptology - EUROCRYPT '96, LNCS 1070, pp. 143154, Springer-Verlag, 1996.

22. A. May. Computing the RSA secret key is deterministic polynomial time equivalent to factoring. Advances in Cryptology - CRYPTO '04, LNCS 3152, pp. 213-219, Springer-Verlag, 2004.

23. M. Michels and M. Stadler. Efficient convertible undeniable signature schemes. Selected Areas in Cryptography - SAC '97, pp. 231-244, Springer-Verlag, 1997.

24. W. Ogata, K. Kurosawa and S.-H. Heng. The security of the FDH variant of Chaum's undeniable signature scheme. The full version of this paper. Available from the Cryptology ePrint Archive, http://www.iacr.org/.

25. T. Okamoto and D. Pointcheval. The gap-problems: a new class of problems for the security of cryptographic schemes. Public Key Cryptography - PKC '01, LNCS 1992, pp. 104-118, Springer-Verlag, 2001.

26. D. Pointcheval and J. Stern. Security proofs for signature schemes. Advances in Cryptology - EUROCRYPT '96, LNCS 1070, pp. 387-398, Springer-Verlag, 1996. 